\title{
Correlation of waist circumference with metabolic syndrome in females attending gynaecology OPD at a tertiary care centre
}

\author{
Poonam Mathur, Pooja Mathur*, Shambhavi Soni
}

Mahatma Gandhi Memorial Medical College and Maharaja Yashwantrao Hospital, Indore, Madhya Pradesh, India

Received: 08 January 2015

Accepted: 17 February 2015

\author{
*Correspondence: \\ Dr. Pooja Mathur, \\ E-mail: poojamathur2604@gmail.com
}

Copyright: (C) the author(s), publisher and licensee Medip Academy. This is an open-access article distributed under the terms of the Creative Commons Attribution Non-Commercial License, which permits unrestricted non-commercial use, distribution, and reproduction in any medium, provided the original work is properly cited.

\begin{abstract}
Background: The metabolic syndrome is an aging-related cluster of metabolic disorders associated with excess adiposity, and plausibly mechanistically linked. The primary goal of recognizing an individual as having the metabolic syndrome was to identify persons at heightened risk for cardiovascular disease (CVD). Secondarily, by design, the diagnosis also helped identify individuals with high risk for other endocrinal imbalance if they did not already have it. So this study was carried out to identify the co-relation between waist circumference and other risk factors with metabolic syndrome and to ascertain their significance.

Methods: This is a prospective observational study conducted in Out Patient Department (OPD) of a tertiary care centre with a study population of 50 patients attending OPD with any gynaecological problem having waist circumference $>85 \mathrm{~cm}$.

Results: Twenty eight patients $(56 \%)$ had metabolic syndrome depicting a significant co-relation $(\mathrm{p}$ value $<0.05)$. Thus, increase in WC more than $85 \mathrm{~cm}$ is directly proportional to the prevalence of metabolic syndrome.

Conclusions: We conclude that Waist circumference is directly related to prevalence of metabolic syndrome which is on increase due to sedentary life style. Such patients are also prone to hormonal imbalance, specifically hypothyroidism.
\end{abstract}

Keywords: Metabolic syndrome, Waist circumference, Hypothyroidism, Obesity

\section{INTRODUCTION}

The metabolic syndrome is an aging related cluster of metabolic disorders associated with excess adiposity, and plausibly mechanistically linked. It frequently develops as the prodromal to the development of overt type 2 diabetes mellitus (T2DM). Over the years, the metabolic syndrome has had various names such as Syndrome X, Cardio metabolic syndrome, Cardiovascular dysmetabolic syndrome, Insulin-Resistance syndrome, Metabolic syndrome (MetS), Beer Belly syndrome, Reaven's syndrome.

\section{Guidelines \& Classification}

Definitions of metabolic syndrome vary. Based on the World Health Organization (WHO) guidelines, the presence of three or more of the following in the same individual indicate metabolic syndrome ${ }^{1}$ :

1. Hypertension/elevated blood pressure - A blood pressure of 130/85 $\mathrm{mm} \mathrm{Hg}$ or higher is a sign of hypertension - the medical term for high blood pressure.

2. Being overweight or obese/excess body fat, particularly around the waist. 
Ethnicity-based differences and the urgent need for a single universally accepted diagnostic tool led to a new definition by the International Diabetes Federation (IDF).

Central adiposity is used as the measurement criterion for the prevalence of metabolic syndrome as it is the central component in all different ethnic groups and can easily be diagnosed with a tape measure. ${ }^{2,3}$

A waistline of $102 \mathrm{~cm}$ or more for men and 85 $\mathrm{cm}$ or more for women is one of the potential indicators of metabolic syndrome. ${ }^{2,3}$

\section{Elevated blood sugar}

A fasting blood glucose (sugar) level greater than $100 \mathrm{mg} / \mathrm{dl}$ hints at abnormal glucose metabolism.

\section{An imbalance in blood fats:}

Both triglyceride levels above $150 \mathrm{mg} / \mathrm{dl}$ and high density lipoprotein level (HDL) - the "good" cholesterol - less than 40-50 mg/dl increase the risk for cardiovascular disease.

Having one indicator of metabolic syndrome increases the risk of developing others. The primary goal of recognizing an individual as having the metabolic syndrome was to identify persons at heightened risk for Cardiovascular Disease (CVD). Secondarily, by design, the diagnosis also helped identify individuals with high risk for diabetes if they did not already have it.

The Adult Treatment Panel III (ATP III), representing the National Cholesterol Education Program (NCEP), published their initial definition of the Meets in 2001 . $^{4}$ As indicated in the ATP III document, its primary purpose was somewhat different from that of the WHO report in that its focus was not on diabetes, but instead to update clinical guidelines for cholesterol testing and management. In addition, a major thrust of this third report by the NCEP was to "focus on primary prevention in persons with multiple risk factors." With these goals in mind, the ATP III introduced the MetS as "multiple, interrelated factors that raise CVD risk." The panel believed that the MetS increased CVD risk at any given low-density lipoprotein cholesterol (LDL-C) concentration, and should be a secondary target of therapy in cholesterol management. Similar to the WHO, the ATP III goal for establishing criteria for the MetS was to identify individuals at special risk for CVD, and to institute intensified lifestyle changes to mitigate these risks. In contrast to the WHO, the ATP III did not consider direct evidence of insulin resistance necessary to make a diagnosis of the MetS., ${ }^{2,3}$
However, there are two more substantive differences between the two organizations in that the ATP III no longer lists micro albuminuria as one of the possible diagnostic criteria, and abdominal obesity, as assessed by measuring waist circumference (WC), is the only acceptable index of excess adiposity. ${ }^{2,3,5}$

Fat distribution may be at least as important as overall fatness in determining risk factor clustering. Many studies use waist circumference as an index of central obesity. In the IRAS study, a large waist circumference was the key predictor of metabolic syndrome; Out of 714 subjects free of metabolic syndrome at baseline, 139 developed metabolic syndrome over 5 years of observation. 5 Patients with MetS are also prone to hormonal imbalance. Dyslipidemia (a high TG and low HDL-C concentration), hyperglycemia, and hypertension are independent factors that directly increase risk of CVD. The relationship between excess adiposity and CVD risk is not the same. At the simplest level, there are substantial numbers of overweight/obese individuals who do not display the components used to make a diagnosis of the MetS. Being overweight/obese simply increases the probability that an individual will become glucose intolerant, dyslipidemic, and hypertensive, and the linchpin between excess adiposity and the remaining components of the various definitions of the MetS is largely a consequence of the adverse effect of being overweight/obese on insulin sensitivity .

This point of view is consistent with the results of the recent study of Ninomiya et al., showing that abdominal obesity, as defined by the ATP III, was the only one of their five variables not statistically associated with the development of either CVD or stroke in an analysis of the NHANES III data. The authors suggested that this finding "may reflect an indirect effect of high WC through other components of the syndrome." Consequently, this section will examine the relationship between excess adiposity, insulin resistance, and the diagnosis of the MetS.

\section{METHODS}

This study was a prospective observational study conducted in an outpatient department of a tertiary care centre with a study population of 50 patients all of which were attending OPD with any gynecological problem and having waist circumference $>85 \mathrm{~cm}$.

\section{Inclusion Criteria}

1. Patient should have waist circumference $>85 \mathrm{~cm}$

\section{Exclusion Criteria}

1. Patient who is pregnant or in puerperal stage.

2. A known case of diabetes or hypertension.

3. A known case of thyroid disorder. 
4. Patient on any type of hormonal treatment like oral contraceptive or hormonal replacement therapy.

Patient not fulfilling above criteria's were excluded from study.

Date was obtained by questionnaires on personal interview, complaints, family history, Personal history, Obstetric history Contraception, life style pattern, Examination findings and Investigations.

Examination included Waist circumference, Height, Weight, Blood Pressure, Pulse, Chest Auscultation.

Investigations Included:

$\begin{array}{ll}- & \text { Serum FBS } \\ - & \text { Serum TSH } \\ - & \text { Serum T3 } \\ - & \text { Serum T4 } \\ - & \text { Serum TG } \\ - & \text { Serum HDL }\end{array}$

BMI was calculated as weight in $\mathrm{kg}$ divided by height $\left(\mathrm{kg} / \mathrm{m}^{2}\right)$ and categorized as $20-25,26-30,31-35$ and $36-$ 40.

\section{RESULTS}

From the study population 28 patients (56\%) had metabolic syndrome. Increase of WC more than $85 \mathrm{~cm}$ is directly proportional to the prevalence of metabolic syndrome. Out of 28 patients having metabolic syndrome, 14 patients $(50 \%)$ were having thyroid disorder (hypothyroidism). Out of the study population 34 patients $(68 \%)$ had sedentary life styles and 22 patients $(44 \%)$ of these 34 had metabolic syndrome. Eight patients $(16 \%)$ were doing regular exercises and 4 (50 \%) were having metabolic syndrome. Out of 50 patients studied 12 patients $(24 \%$ ) had family history of diabetes mellitus and out of this 8 patients $(33.33 \%)$ had metabolic syndrome. Eight (16\%) patients had a family history of hypertension and out of this 4 patients $(50 \%)$ had metabolic syndrome. Out of 50 patients studied 8 patients had family history of thyroid disorder and out of this 2 patients $(25 \%)$ had metabolic syndrome. Patients having pure vegetarian diet were at small risk of having metabolic syndrome as compared to non-vegetarians.

\section{DISCUSSION}

Though the pathogenesis of the metabolic syndrome and its components is complex, abdominal obesity is a key causative factor. Despite the importance of obesity in the model, we should remember that patients of normal weight can also be insulin resistant. Those are called metabolically obese, normal-weight individuals, typically having increased amount of visceral adipose tissue. According to some theories, with increase in visceral adipose tissue, a higher rate of flux of adipose tissue- derived free fatty acids to the liver through the splanchnic circulation would be expected, while increases in abdominal subcutaneous fat could release lipolysis products into the systemic circulation and avoid more direct effects on hepatic metabolism., ${ }^{3,4}$

Table 1: Distribution of study population on the basis of waist circumference.

\begin{tabular}{|lll|}
$\begin{array}{l}\text { Waist } \\
\text { circumference } \\
\text { (In cm) }\end{array}$ & $\begin{array}{l}\text { No. } \\
\text { of } \\
\text { cases }\end{array}$ & Percentage \\
\hline$>85-90$ & 18 & $36 \%$ \\
\hline $91-95$ & 14 & $28 \%$ \\
\hline $96-100$ & 10 & $20 \%$ \\
\hline $101-105$ & 0 & $0 \%$ \\
\hline $111-115$ & 2 & $4 \%$ \\
\hline
\end{tabular}

Table 2: Relation of BMI (kg/m2) with metabolic syndrome.

\begin{tabular}{|ll|}
\hline BMI & $\begin{array}{l}\text { \% of Cases having } \\
\text { MetS }\end{array}$ \\
\hline $20-25$ & $66.66 \%$ \\
\hline $26-30$ & $40 \%$ \\
\hline $31-35$ & $80 \%$ \\
\hline $36-40$ & $100 \%$ \\
\hline
\end{tabular}

Table 3: Correlation between metabolic syndrome and other parameters studied.

\begin{tabular}{|lll|}
\hline Parameters & $\begin{array}{l}\text { Standard 'P' } \\
\text { value } \\
\text { Taken from } \\
\text { study by } \\
\text { Hollenbeck }\end{array}$ & $\begin{array}{l}\text { Observed 'P' } \\
\text { value } \\
\text { Study }\end{array}$ \\
\hline $\begin{array}{l}\text { Waist } \\
\text { Circumference }\end{array}$ & $<0.49$ & $<0.001$ \\
\hline $\begin{array}{l}\text { Thyroid } \\
\text { dysfunction }\end{array}$ & $\leq 0.008$ & $<0.001$ \\
\hline $\begin{array}{l}\text { Fasting Blood } \\
\text { Sugar }\end{array}$ & $<0.001$ & $<0.001$ \\
\hline Triglyceride & $<0.07$ & $<0.06$ \\
\hline $\begin{array}{l}\text { High Density } \\
\text { Cholesterol }\end{array}$ & $<0.001$ & $<0.05$ \\
\hline Blood Pressure & $<0.005$ & $<0.001$ \\
\hline
\end{tabular}

Hypertriglyceridemia is an excellent reflection of the insulin resistant condition and is one of the important criteria for diagnosis of the metabolic syndrome. The other major lipoprotein disturbance in the metabolic syndrome is a reduction in HDL cholesterol. This reduction is a consequence of changes in HDL composition and metabolism. In the presence of hypertriglyceridemia, a decrease in the cholesterol content of HDL results from decrease in the cholesteryl ester content of the lipoprotein core with variable 
increases in triglycerides. In addition to HDL, the composition of LDL is also modified in a similar way. In fact, with fasting serum triglycerides > $2.0 \mathrm{mmol} / \mathrm{L}$, almost all patients have a predominance of small dense LDL. This change in LDL composition is attributable to relative depletion of unesterified and esterified cholesterol, and phospholipids, with either no change or an increase in LDL triglyceride. In some studies, this alteration in LDL composition is an independent risk factor for cardiovascular disease. However, more often this association is not independent, but related to the concomitant changes in other lipoproteins and other risk factors.

In this study we have emphasized on the presence of aforementioned risk factors and to stop them at that point only. For solving the purpose, life style modifications are must. As the prevalence of sedentary lifestyle is increasing day by day prevalence of metabolic syndrome is on rise, which is itself a risk factor for bag full diseases.

In this study it was found that out of 50 cases studied having WC more than $85 \mathrm{~cm}$, fifty six percent $(56 \%)$ have metabolic syndrome and out of these people $50 \%$ had thyroid disorders.

This data is sufficient to extract that WC is directly related to metabolic syndrome, as WC increases risk of having metabolic syndrome increases.

Also patients having metabolic syndrome have a higher possibility of developing hormonal disorders but as this study is strictly concerned with thyroid disorder, so finding out prevalence of thyroid disorder shows that out of $56 \%$ population having metabolic syndrome $50 \%$ have thyroid disorder.

Table - 3 shows discrepancy in value of significance for different parameters in relation to metabolic syndrome. This difference is due to difference in study population. We had taken only non-pregnant obese females into consideration while standard studies were done on both genders. The difference may also be due to study population and study area.

\section{Life style}

In the present study population $68 \%$ had sedentary life style. This shows that ignorance leads to syndrome \& exercise is must. Study shows that a vicious cycle runs in a obese sedentary female due to hormonal imbalance which leads to insulin resistance and the female gains weight day by day and ultimately becomes a part of the syndrome well known as metabolic syndrome.

\section{Genetic basis}

Present study shows a correlation of DM with metabolic syndrome in patients having family history of DM, an already proved issue. At the same time history of hypertension or any thyroid dysfunction also have a strong correlation with metabolic syndrome when it is present in one or two parents.

These results may be due to differences in the study populations in terms of age, sex, race, definition of mild hypothyroidism, and differences in the duration of follow-up.

\section{CONCLUSIONS}

Our study has the mere purpose to find out the prevalence of metabolic syndrome among obese non pregnant females coming to OPD at tertiary centres for other gynecological problems. We conclude that WC is directly related to prevalence of metabolic syndrome which is on increase due to sedentary life style. Patients who are obese may have hormonal imbalance. Among the study population $56 \%$ have metabolic syndrome \& among these $56 \%$ syndromic patients, $50 \%$ have thyroid disorder (hypothyroidism).

The results of the present study also show that there is a significant relationship between thyroid function and cardiovascular risk factors, such as BP, total cholesterol, TG, HDL-C and fasting glucose. Also, higher levels of TSH may predict the MetS in Indian population. In our study population $68 \%$ had sedentary life style. This shows that ignorance leads to the syndrome \& regular exercises are must. This study shows a correlation of DM with metabolic syndrome in patients having family history of DM, an already proved issue.

\section{Funding: No funding sources Conflict of interest: None declared \\ Ethical approval: Not required}

\section{REFERENCES}

1. Alberti KG,Zimmet PZ. Definition, diagnosis and classification of diabetes mellitus and its complications. Part 1: diagnosis and classification of diabetes mellitus provisional report of a WHO consultation. Diabet Med. 1998;15(7):539-53.

2. Tsigos C, Chrousos GP. Differential diagnosis and management of Cushing's syndrome. Annu Rev Med. 1996;47:443-61.

3. Friedman TC, Mastorakos G, Newman TD, Mullen NM, Horton EG, Costello R, et al. Carbohydrate and lipid metabolism in endogenous hypercortisolism: shared features with metabolic syndrome $\mathrm{X}$ and NIDDM. Endocr J. 1996;43(6):645-55.

4. Executive Summary of The Third Report of The National Cholesterol Education Program (NCEP) Expert Panel on Detection, Evaluation, And Treatment of High Blood Cholesterol In Adults (Adult Treatment Panel III). Jama. 2001;285(19):2486-97. 
5. Facchini F, Hollenbeck CB, Chen YN, Chen YD, Reaven GM. Demonstration of a relationship between white blood cell count, insulin resistance, and several risk factors for coronary heart disease in women. J Intern Med. 1992;232(3):267-72.
DOI: $10.5455 / 2320-1770$. ijrcog20150411

Cite this article as: Mathur $\mathrm{P}$, Mathur $\mathrm{P}$, Soni S. Correlation of waist circumference with metabolic syndrome in females attending gynaecology OPD at a tertiary care centre. Int J Reprod Contracept Obstet Gynecol 2015;4:344-8. 